\title{
Metastatic Lobular Carcinoma of the Breast in Pleural Fluid: Diagnostic Pitfall for Mesothelial Cells
}

\author{
Yoon Mi Jin ${ }^{1}$, Sharon L. Hirschowitz ${ }^{2}$ and Sophia K. Apple ${ }^{*}, 2$ \\ ${ }^{I}$ Department of Pathology, Seoul Soon Chun Hyang University Hospital, Seoul Korea \\ ${ }^{2}$ Department of Pathology, David Geffen School of Medicine, University of California at Los Angeles, Los Angeles, \\ California, USA
}

\begin{abstract}
Cytomorphologic patterns of breast carcinoma in effusion include three-dimensional groups of tightly packed clusters for invasive ductal type and single files with cytoplasmic signet ring-like cells for invasive lobular type. A mesothelial-like cell pattern has been described for metastatic lobular carcinoma in pleural fluid. We report a case of a patient with a remote history of lobular carcinoma of the breast who presented with hypocellular pleural effusion with the mesothelial-like cell pattern. Without the previous clinical history and the help of marker studies, the mesothelial-like cell pattern of metastatic lobular carcinoma to pleural fluid may be difficult to distinguish from benign reactive/atypical mesothelial cells.
\end{abstract}

Keywords: Metastatic lobular carcinoma, breast, pleural fluid.

\section{INTRODUCTION}

Lobular carcinoma is a type of mammary carcinoma characterized by multifocality and cytomorphologic features of small, relatively uniform, bland appearing tumor cells with single file pattern without stromal desmoplasia. Lobular carcinoma of the breast has a greater propensity to metastasize to the leptomeninges, peritoneal surfaces, retroperitoneum, gastrointestinal tract, reproductive organs and bone in contrast to the ductal carcinoma, which shows common metastasis to the lung, liver, brain and pleural cavity [1]. Infiltrating lobular carcinoma is one of the most difficult types to identify in fine-needle aspiration as well as body fluid cytology because of the innocuous appearance of the tumor cells and the lack of cohesive clusters, thus being a frequent cause of false negative diagnosis on cytology especially when the specimen is paucicellular [2-4]. It is also known that when the lobular carcinoma metastasizes to the body fluid, the distinction between metastatic carcinoma cells and mesothelial cells can be very difficult. In this report, we demonstrate a case of metastatic lobular carcinoma in pleural fluid mimicking benign and reactive mesothelial cells which could have lead to false negative diagnosis if we had no knowledge of clinical history, clinical presentation and without the help of ancillary studies.

\section{CASE REPORT}

A 60-year-old woman was admitted to the hospital due to dyspnea and shortness of breath worse on exertion, for a few months. She had a history of breast cancer from the right side 16 years ago and had undergone lumpectomy with axillary lymph node dissection, chemotherapy and radiation

*Address correspondence to this author at the Department of Pathology, David Geffen Medical Center, 10833 Le Conte Ave CHS 1P-244, Los Angeles, CA 90098-1732, USA; Tel: 310-825-9288;

Fax: 310-267-2058; E-mail: sapple@mednet.ucla.edu therapy. The tumor was reported as an invasive lobular carcinoma, classic type with 4 of 11 lymph nodes involved. Her initial chest X-ray on admission showed near-complete opacification of the right hemithorax, most likely due to large right pleural effusion and atelectasis. She then had a follow up chest CT which revealed large right tension hydrothorax, suspicious for malignant pleural effusion. Subsequent MRI showed a large right pleural effusion suggestive of pleural carcinomatosis and multiple rib metastases involving the right $5^{\text {th }}, 7^{\text {th }}$, left $5^{\text {th }}$ and $6^{\text {th }}$ ribs. Multiple lymph nodes including right cardiophrenic angle, right internal mammary and right periaortic lymph nodes were enlarged concerning for metastasis clinically. She has undergone thoracentesis and a pleural fluid specimen of 800 yellow cloudy material was sent for cytologic evaluation. Papanicolaou (PAP), May-Grunewald-Giemsa (MGG) stains and cell block were made.

\section{Cytologic Findings}

The pleural fluid cytology specimen was hypocellular composed of small lymphoid cells, histiocytes and benign mesothelial cells and a few dyshesive and loosely cohesive atypical cells (Figs. 1, 2). Some of the atypical cells were singly scattered or loosely aggregated with three or more cells with rare linear pattern, mimicking reactive mesothelial cells. These atypical cells had slightly irregular nuclei with occasional prominent nucleoli and high nuclear to cytoplasmic ratio. There were occasional cells with vacuolization and peripheral lucent zone in the cytoplasm (Fig. 3). While the cells showed two dimensional, flat, and loosely arranged sheets of cells as seen in reactive mesothelial cells, these cells lacked two-tone cytoplasm; dense inner cytoplasm and paler outer layer, cytoplasmic windows between cells, knobbly cytoplasmic borders, and peripheral halos. The cell block section showed more cellular material, admixed with acute inflammatory cells, lymphocytes, mesothelial cells and scattered dispersed, 
dyshesive, mildly atypical larger cells (Fig. 4). These larger cells had plasmacytoid features with eccentrically placed nuclei and were monomorphic in appearance. No evidence of intracytoplasmic mucin targetoid vacuoles was noted in the cytoplasm in the cell block section. The differential diagnosis included metastatic lobular carcinoma and reactive/atypical mesothelial cell proliferation.

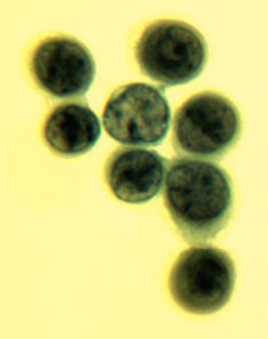

Fig. (1). Metastatic lobular carcinoma in pleural fluid showing loosely aggregated mildly atypical cells (PAP stain, oil magnification).

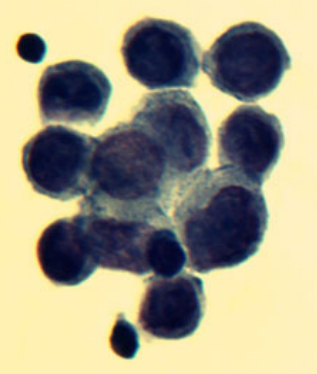

Fig. (2). Metastatic lobular carcinoma in pleural fluid showing loosely aggregated atypical cells in single or mesothelial-like pattern but without cytoplasmic knobby processes and window (MGG stain, oil magnification).

\section{Immunohistochemical Findings}

Immunohistochemical stains were performed on the cell block of the pleural fluid.

The cell block was fixed in $10 \%$ neutral-buffered formalin prior to immunohistochemical stains. The immunohistochemical staining was performed on the Ventana Benchmark XT system (Tucson, AZ) with iView DAB detection. The antibodies used were: BerEP4, B72.3, CD15, MOC-31, Calretinin, and estrogen receptor(ER). The immunostains performed on the cell block from the pleural fluid were positive for B72.3 (10-15\% of the cells) and ER (10-15\% of the cells) and negative for BerEP4, CD15, and
MOC-31. Scattered cells were positive for Calretinin representing background mesothelial cells (Figs. 5, 6). We retrieved the slides and blocks from her original tumor 16 years ago and performed breast biomarkers. Her original tumor was reported "ER positive" without quantification because it was a ligand assay test. Immunohistochemical stains for ER and PR on the original tumor block showed strong positivity ( $95 \%$ with $3+$ intensity) for both markers and Her-2/neu was not overexpressed with a score of $1+$. FISH Her-2/neu test was also performed on the original tumor section and revealed no amplification. Cytomorphology of the invasive lobular carcinoma from the original tumor was similar to atypical cells seen in the pleural fluid. We concluded the atypical cells in the pleural fluid as metastatic lobular carcinoma by the cytologic findings, clinical suspicion and immunostain results.

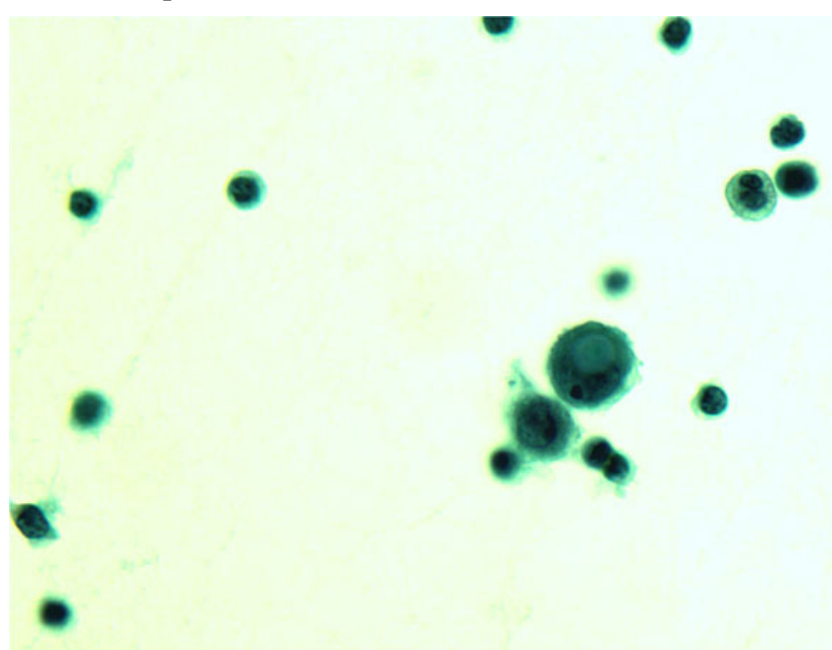

Fig. (3). Rare cytoplasmic vacuole with targetoid mucin is seen (PAP stain, oil magnification).

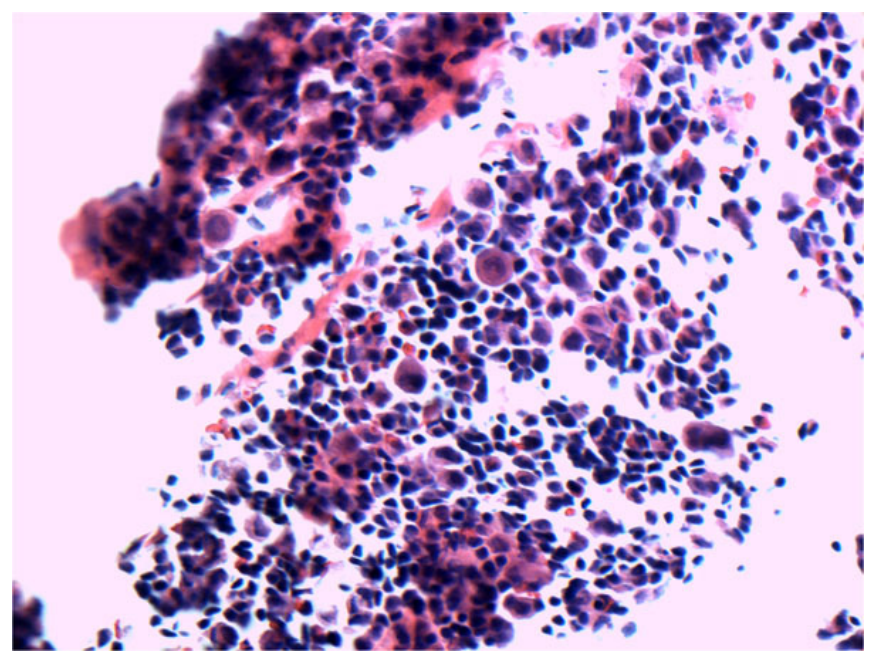

Fig. (4). Cell block section of metastatic lobular carcinoma in pleural fluid. Scattered dispersed single cells with abundant cytoplasm are seen in the inflammatory background (H\&E stain x400 magnification).

\section{DISCUSSION}

The metastasis of lobular carcinoma in pleural fluid is rare and very difficult to detect due to tumor cells resembling reactive mesothelial cells [1]. The inherent cytologic 
characteristics of lobular carcinoma cells as small size and bland appearance especially in scanty cellular material in cavity fluid may lead to false-negative diagnosis. This case highlights the importance of performing immunostains when the pleural fluid specimens show reactive/atypical mesothelial-like cells in the setting of a clinical history suspicious for metastatic lobular carcinoma. There are limited reported cases in literature that describes metastatic lobular carcinoma to pleural effusion. Lobular carcinoma is reported to have predilection for peritoneal spread rather than pleural cavity $[2,3]$. The largest series of metastatic carcinomas in effusions was reviewed by Antic et al., [4] with metastatic lobular carcinoma in only 2 out of 249 cases, $(0.8 \%)$ in pleural fluid and $21.6 \%$ in peritoneal fluid.

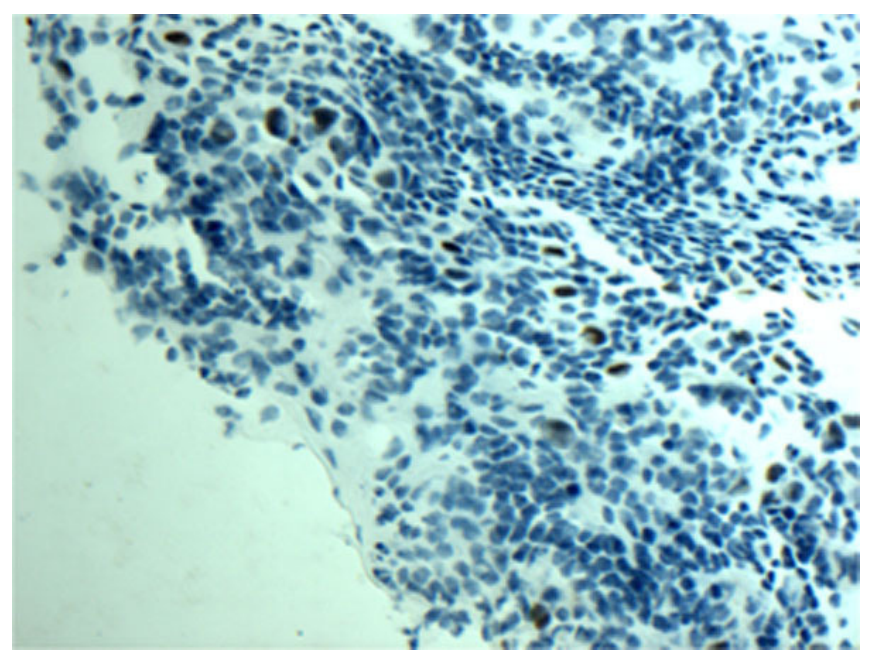

Fig. (5). Cell block section of metastatic lobular carcinoma in pleural fluid. Scattered dispersed single cells with abundant cytoplasm are positive with ER stain (Immunohistochemical stains x400 magnification).

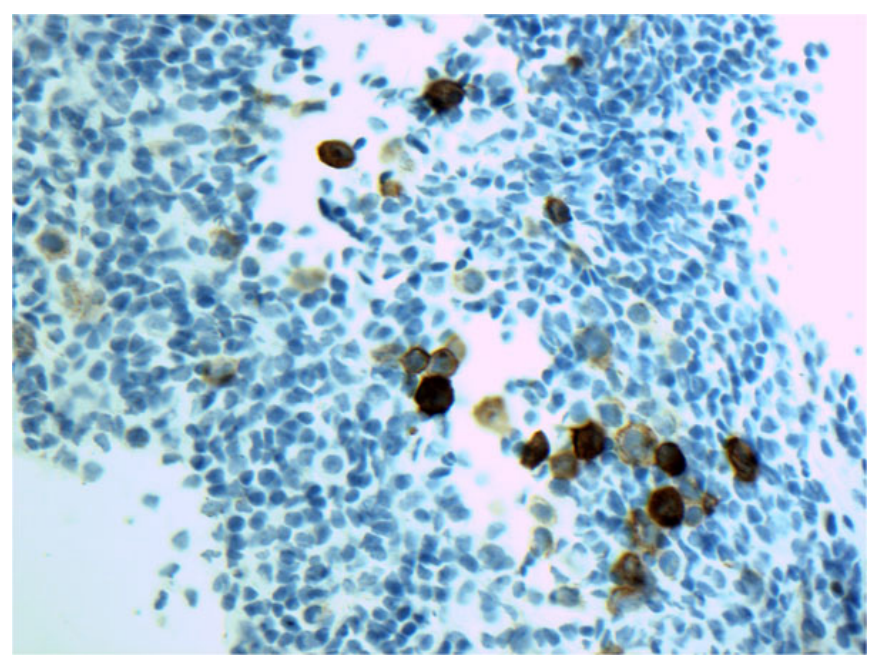

Fig. (6). Cell block section of metastatic lobular carcinoma in pleural fluid. Scattered dispersed single cells with abundant cytoplasm are positive with B72.3 stain (Immunohistochemical stains x400 magnification).

The cytologic features of invasive lobular carcinoma in fine needle aspiration cytology are relatively well described in the literature. In the classic type, the specimen is usually low to moderately cellular, composed predominantly of small cells which are dyshesive with single file arrangement.
The cells are monomorphic with eccentric located and vesicular to hyperchromatic nuclei and have scant to moderate amounts of cytoplasm. Intracytoplasmic lumina, represented by a central bluish or metachromatic dot-like structure surrounded by a clear halo, which can be best seen in Diff-Quick- and MGG-stained smears and signet ring cell features, if present, are helpful to diagnose lobular carcinoma.

Several cytologic patterns of breast lobular carcinoma in body fluid have been described, which includes cannonball or three-dimensional cluster pattern, single-cell or mesothelial-like cell pattern [4-7]. The single or mesotheliallike cell pattern, which is characterized by cell monotony, eccentric nuclei with plasmacytoid features, secretory vacuoles and intracytoplasmic lumina, is characteristic of metastatic lobular carcinoma although it can be also found in $6.0 \%$ of metastatic ductal carcinoma [4]. However, unlike in metastatic lobular carcinoma, most of ductal carcinoma cells show nuclear pleomorphism with overt malignant features. Pleomorphic lobular carcinoma in pleural fluid has been described by Monaco S et al., [8]. The differential diagnosis of pleomorphic lobular carcinoma in the body fluid includes not only reactive/atypical mesothelial cells but also malignant epithelioid mesothelioma.

Danner et al., [9] also emphasized the characteristic single cell appearance with hyperchromatic nuclei, scanty cytoplasm and conspicuous nucleoli and their single file configuration and bull's-eye arrangement as basic patterns in effusions. The bland cytologic characteristics of tumor cells make the cytologic distinction between tumor cells and reactive mesothelial cells very difficult. The mesothelial cells are known to have distinct cytologic features, such as two-tone cytoplasm (dense inner cytoplasm and paler outer layer), windows between mesothelial cells, and peripheral halos representing the microvillous border [9]. Two dimensional, flat, and loosely arranged sheets of cells are the pattern frequently seen in reactive mesothelial cells [10]. Mesothelial cells are also known to have a variety of reactive changes in the setting of various diseases.

Metastatic lobular carcinoma cells in body fluid are easy to misdiagnose as reactive mesothelial cells because of the relative bland appearing cytomorphology and the resultant difficulty to perceive foreignness in fluid cytology. Although the metastatic lobular carcinoma cells in the present case seem to be arranged singly or in more loosely aggregated small clusters with rare linear pattern, the morphologic differences between tumor cells and mesothelial cells are so subtle that it was very difficult to discern the two types of cells without the help of ancillary studies such as immunostains.

Several immunostain markers are helpful to differentiate metastatic adenocarcinoma from reactive/atypical mesothelial cells. Carcinoembryonic antigen (mCEA), B72.3, BerEP4, Leu M1 (CD 15), and MOC-31 are the commonly used epithelial markers whereas calretinin, D2-40, WT-1, HBME1 , and cytokeratin $5 / 6$ can be helpful to support a mesothelial origin $[11,12]$. Of those epithelial cell markers, MOC-31 is reported to be a superior marker to differentiate metastatic lobular carcinoma from the mesothelial cells since it was reported to be positive in $89 \%$ of metastatic lobular carcinoma whereas Ber-EP4 showed positivity in only 
$27.5 \%$ [13]. Our case revealed negativity for MOC-31 in the tumor cells. A few positive cells for ER (estrogen receptor), and B72.3 supported the presence of metastatic lobular carcinoma in our case.

Most patients with a malignant effusion have a known history. If a positive effusion is the first sign of an unsuspected malignancy of lobular carcinoma of the breast, false-negative diagnosis may result. Subtle clues among the cytologic features may include singly scattered small atypical cells with occasional linear or single file pattern and intracytoplasmic lumina, if present, may be helpful findings in pleural effusions in women. In conclusion, considering the difficulty of appreciating a foreign population in cases of metastatic lobular carcinoma in body fluid, it is of the utmost importance to have a high index of suspicion accompanied by meticulous cytologic inspection and initiation of further ancillary studies, especially in patients with a history of breast carcinoma.

\section{ACKNOWLEDGEMENTS}

None declared.

\section{CONFLICT OF INTEREST}

None declared.

\section{REFERENCES}

Ashton PR, Hollingsworth AS Jr, Johnston WW. The cytopathology of metastatic breast cancer. Acta Cytol 1975; 19: 16.
[2] Borst MJ, Ingold JA. Metastatic patterns of invasive lobular versus invasive ductal carcinoma of the breast. Surgery 1993; 114: 641-2.

[3] Lamovec J, Bracko M. Metastatic pattern of infiltrating lobular carcinoma of the breast: an autopsy study. J Surg Oncol 1991; 17: 251-7.

[4] Antic T, Gong Y, Sneige N. Tumor type and singlecell/mesothelial-like cell pattern of breast carcinoma metastases in pleural and peritoneal effusions. Diagn Cytopathol Early View 2010; 1-5. (22 NOV 2010 on line DOI: 10.1002/dc.21563).

[5] Danner DE, Gmelich JT. A comparative study of tumor cells from metastatic carcinoma of the breast in effusions. Acta Cytol 1975; 19: $509-18$.

[6] Bedrossian CWM. Malignant effusions: A multimodal approach to cytologic diagnosis. New York: Igaku-Shoin. 1994, p. 120.

[7] Tao LC. Cytopathology of malignant effusion. ASCP Theory Practice Cytopathol 1995; 6: 89 .

[8] Monaco SE, Dabbs DJ, Kanbour-Shakir A. Pleomorphic lobular carcinoma in pleural fluid:diagnostic pitfall for atypical mesothelial cells. Diagnostic Cytopathol 2008; 36: 657-61.

[9] DeMay RM. The art and science of cytopathology:Exfoliative cytology. Chicago: ASCP Press. 1996, p. 462.

[10] Cakir E, Demirag F, Aydin M, Unsal E. Cytopathologic differential diagnosis of malignant mesothelioma, adenocarcinoma and reactive mesothelial cells: A logistic regression analysis. Diagn Cytopathol 2009; 37: 4-10.

[11] Pereira TC, Saad RS, Liu Y, Silverman JF. The diagnosis of malignancy in effusion cytology: A pattern recognition approach. Adv Anat Pathol 2006; 13: 174-84.

[12] Kim JH, Kim GE, Choi YD, et al. Immunocytochemical panel for distinguishing between adenocarcinomas and reactive mesothelial cells in effusion cell blocks. Diagn Cytopathol 2009; 37: 258-61.

[13] Pai RK, West RB. MOC-31 exhibits superior reactivity compared with Ber-EP4 in invasive lobular and ductal carcinoma of the breast: a tissue microarray study. App Immunohistochem Mol Morphol 2009; 17: 202-6. 